\title{
Therapeutic Plasma Exchange: An Indispensable Therapy for Severe Neurological Conditions
}

\author{
Rodica Balasa* \\ Neurology Department, George Emil Palade University of Medicine, Pharmacy, Science, and Technology of Targu \\ Mures, Romania
}

Received: 31 January 2020 / Accepted: 24 February 2020

Therapeutic plasma exchange (TPE) is the most frequent therapeutic apheresis procedure used to remove the plasma, together with its high-molecular-weight agents such as immune complexes, antibodies, complement components, cytokines, different toxins and cryoglobulins, as well as to return of the majority of cellular components to the patients [1]. In the hands of an experienced specialist, TPE has been found by the American Academy of Neurology to be a very important and safe tool that can improve neurological disability in patients with numerous disorders [2].

Numerous recent reviews and research articles have shown the benefit of TPE in different neurological diseases in both adults and children $[3,4]$. In neurological diseases such as acute inflammatory demyelinating polyradiculoneuropathy (Guillain-Barré syndrome; GBS), chronic inflammatory demyelinating polyradiculoneuropathy, myasthenia gravis (MG) and paraneoplastic encephalopathies, TPE is accepted as firstline therapy, either as a primary standalone treatment or combined with other treatments. TPE is accepted as second-line therapy, usually associated with other modes of treatment, for numerous other neurological diseases, including acute disseminated encephalomyelitis, chronic focal encephalitis (Rasmussen's encephalitis), Lambert-Eaton myasthenic syndrome, Sydenham's chorea, natalizumab-associated progressive multifocal leukoencephalopathy, multiple sclerosis and neuromyelitis optica (NMO, or Devic's disease) [5].

The effect of TPE in neurological cases may be measured in clinical terms (motor deficit, muscle power grading, lack of mechanical ventilation, breathing efficiency or gait improvement) and in paraclinical assessments (antibody levels).

TPE is most frequently used in peripheral nervous system autoimmune diseases such as GBS, accounting for approximately $70 \%$ of all patients benefiting from TPE in a specialised department in India [1]. In axonal forms of GBS, TPE has been found to be the most effective therapy, more effective than intravenous immunoglobulins [5]. The maximum benefit is obtained if TPE is used within the first seven days of the GBS disease course.

MG is an autoimmune disease of the neuromuscular junction, where the titre of antibodies against acetylcholine receptor (Ach-R) is high in more than 70\% of cases. Not only these cases but all MG cases benefit from TPE, mainly during myasthenic respiratory crisis and myasthenia exacerbation. In some patients, this procedure, which rapidly reduces the titre of autoantibodies, may lead to a rebound overproduction of these antibodies against Ach-R. This seems to generate a vulnerability of the producing cells to cytotoxic drugs. Because of this, TPE is frequently associated with therapies that have an immunosuppressive effect, such as prednisone and azathioprine [3, 5-8].

$\mathrm{NMO}$ is an inflammatory demyelinating disease of the central nervous system that affects predominantly the optic nerves and spinal cord and usually follows a relapsing-remitting course. Since the description by Lennon et al. [9] of anti-aquaporin 4 antibodies in the serum of NMO patients, the use of TPE during relapses or for preventing severe relapses has significantly improved clinical outcomes. The immunopathogenesis in NMO is humoral immune-mediated, and as a consequence, the response following TPE is sometimes more profound than that found in other autoimmune diseases. TPE performed as early as possible has led in some cases to the Lazarus effect (immediate dramatic improvement). In some NMO cases, if TPE is performed very late after a relapse onset, severe axonal injury might be stalled [10-13]. 
Paraneoplastic neurological disorders are severe heterogeneous neurological syndromes due to distant immunological effects of malignancy. These neurological disorders often occur before the cancer diagnosis (50\% of cases). For this reason, early recognition, neoplasia workup and treatment are crucial. The essential treatment is mainly early cancer spotting and elimination. TPE together with other immunosuppressant drugs has been reported to provide clinical improvement [14].

Autoimmune encephalitis is a rare group of diseases, and in up to $50 \%$ of cases, patients are found negative for the usual autoimmune antibodies tested. Interestingly, TPE applied in all autoimmune encephalitic cases might improve patient outcomes [15].

TPE is effective in removing different therapeutic proteins from the body. The clearance of natalizumab (a monoclonal antibody used for the treatment of multiple sclerosis) is sometimes needed to treat complications from treatment, such as progressive multifocal leukoencephalopathy (PML). In this encephalopathy, immune reconstitution is the only measure to potentially improve the clinical outcome from PML. TPE accelerates the clearance of natalizumab from the body and could restore the immune function of lymphocytes that show efficacy for PML regression [16].

In some diseases, TPE is considered equally effective as intravenous immunoglobulins. TPE used as immunomodulatory treatment is also cheaper than intravenous immunoglobulins [5]. TPE has become a very safe treatment due to improvements in apheresis machines.

Like any other treatment procedure, TPE has some disadvantages: approximatively the same amount of removed plasma needs to be replaced with albumin or frozen plasma to maintain an optimal intravascular compartment. In many emergency neurological centres, TPE has become standard practice as a rescue therapy and/or maintenance therapy.

\section{CONFLICT OF INTEREST}

None to declare.

\section{REFERENCES}

1. Shreedevi SB, Karkal RN. Therapeutic Plasma Exchange - An Emerging Treatment Modality in Patients with Neurologic and Non-Neurologic Diseases. J Clin Diagn Res. 2017;11(8):EC35EC37.
2. Subcommittee of the American Academy of Neurology. Neurology. Assessment of plasmapheresis: report of the Therapeutics and Technology Assessment. Neurology. 1996;47:840-43.

3. Savransky A, Rubstein A, Rios $\mathrm{MH}$, et al. Prognostic indicators of improvement with therapeutic plasma exchange in pediatric demyelination. Neurology. 2019;93:e2065-e2073.

4. McLeod BC. Therapeutic Plasma Exchange. In: Blood banking and transfusion medicine, Second Edition. Philadelphia, PA: Elsevier publishing. 2009, pp. 738-764.

5. Heatwole C, Johnson N, Holloway R, Noyes K. Plasma exchange versus intravenous immunoglobulin for myasthenia gravis crisis: An acute hospital cost comparison study. J Clin Neuromuscul Dis. 2011;13:85-94.

6. Gajdos P, Chevret S, Toyka K. Plasma exchange for myasthenia gravis. Cochrane database Syst Rev. 2002;(4):CD002275.

7. Kumar R, Birinder SP, Gupta S, Singh G, Kaur A. Therapeutic plasma exchange in the treatment of myasthenia gravis. Indian J Crit Care Med. 2015; 19(1): 9-13.

8. Tombak A, Uçar MA, Akdeniz A, Yilmaz, et al. Therapeutic plasma exchange in patients with neurologic disorders: Review of 63 cases. Indian J Hematol Blood Transfus. 2017;33(1):97105. .

9. Lennon VA, Wingerchuk DM, Kryzer TJ, et al. A serum autoantibody marker of neuromyelitis optica: distinction from multiple sclerosis. Lancet. 2004;364(9451):2106-12.

10. Bălașa R, Maier S, Bajkó Z, Moțățăianu A, Crișan A, Bălașa A. Longitudinally extensive transverse myelitis in neuromyelitis optica: a prospective study of 13 Caucasian patients and literature review. Acta Neurol Belg. 2015;115:635-42.

11. Bălașa R, Bajkó Z, Moțățăianu A, Maier A, Maier S. Neuromyelitis optica associated with painful dystonia: case report and literature review. Acta Neurol Belg. 2015;115:169171.

12. Stoian A, Motataianu A, Bajko Z, Balasa A. Guillain-Barré and Acute Transverse Myelitis Overlap Syndrome Following Obstetric Surgery. The Journal of Critical Care Medicine. 2020;6(1):74-9

13. Kumawat $B L$, Choudhary $R$, Sharma $C M$, Jain D, Hiremath $A$. Plasma Exchange as a First Line Therapy in Acute Attacks of Neuromyelitis Optica Spectrum Disorders. Ann Indian Acad Neurol. 2019;22:389-94

14. Sadeghian H, Department of Neurology, University of Texas Southwestern Medical Center, Dallas, TX, USA

15. Vernino S. Progress in the management of paraneoplastic neurological disorders. Ther Adv Neurol Disord. 2010;3(1):4352.

16. Pandey PK, Agarwal N, Gupta M. Therapeutic plasma exchange as a life-saving therapy in a suspected case of autoimmune encephalitis: A case report from a tertiary health-care center. Asian J Transfus Sci. 2018;12(2):173-5.

17. Khatri BO, Man S, Giovannoni G, et al. Effect of plasma exchange in accelerating natalizumab clearance and restoring leukocyte function. Neurology. 2009;72(5):402-9. 